\title{
Clinical efficacy of montelukast sodium combined with budesonide or combined with loratadine in treating children with cough variant asthma and influence on inflammatory factors in the serum
}

\author{
HAIYAN WEI, WEIHUA LI, ZHEN JIANG, XIAOYING XI and GONGJIAN QI
}

Department of Intensive Care Unit, Xuzhou Children's Hospital, Xuzhou Medical University, Xuzhou, Jiangsu 221006, P.R. China

Received October 12, 2018; Accepted April 19, 2019

DOI: 10.3892/etm.2019.7574

\begin{abstract}
Clinical efficacy of montelukast sodium combined with budesonide or combined with loratadine in children with cough variant asthma was investigated. A retrospective analysis of the medical records of 72 children with cough variant asthma who were treated in Xuzhou Children's Hospital, Xuzhou Medical University from April 2015 to August 2017 was performed and the 72 child patients were divided into two groups: 35 children were treated with montelukast sodium combined with budesonide in Group A, and 37 children were treated with montelukast sodium combined with loratadine in Group B. The clinical efficacy of the two groups was evaluated according to the lung function indexes [forced expiratory volume in the first second (FEV1), ratio of the forced expiratory volume in the first second to the forced vital capacity (FEV1/FVC), the peak expiratory flow (PEF)], the inflammation biomarkers [tumor necrosis factor- $\alpha$ (TNF- $\alpha$ ) and interleukin-4 (IL-4)], the level of eosinophil granulocyte, and the level of $\operatorname{IgE}$ at three time-points: before treatment, the 4th week after treatment, and the 12th week after treatment as well as adverse reactions, recurrence of symptoms, and treatment compliance were recorded. After treatment, the levels of FEV1, FEV1/FVC, PEF, TNF- $\alpha$ and IL-4, eosinophil granulocyte and $\operatorname{IgE}$ in the two groups were significantly improved $(\mathrm{P}<0.05)$. The treatment compliance of Group A was significantly lower than that of Group B $(\mathrm{P}<0.05)$. In conclusion, the method of montelukast sodium combined with budesonide or loratadine are both worthy of clinical promotion because they have equivalent efficacy in the treatment of cough variant asthma to effectively improve the lung function and inflammatory response in patients and both bring less adverse reactions and lower recurrence rate.
\end{abstract}

Correspondence to: Dr Gongjian Qi, Department of Intensive Care Unit, Xuzhou Children's Hospital, Xuzhou Medical University, 18 Sudi North Road, Xuzhou, Jiangsu 221006, P.R. China

E-mail: qi_gongjian1@163.com

Key words: montelukast sodium, atomization inhalation, loratadine, cough variant asthma

\section{Introduction}

Often featuring intensified cough in the morning and at night and receiving no efficacy from antibiotics, cough variant asthma, a special kind of chronic recurrent cough that is characterized by the involvement of a variety of cells and cell components, is one of the most prominent causes of chronic cough in children $(1,2)$. Approximately $30-54 \%$ of cough variant asthma in children with this disease deteriorates further to develop into typical bronchial asthma. With the changing society and living habits, cough variant asthma is showing an increasing incidence, greatly impacting the learning, physical and mental health of children suffering from it $(3,4)$. Therefore, an active clinical treatment is required for children with cough variant asthma.

According to the consensus of experts worldwide, the treatment strategy of cough variant asthma is basically the same as the treatment of bronchial asthma, mainly using montelukast sodium, budesonide and loratadine in today's clinical practice (5-7). Montelukast sodium is a highly specific and selective leukotriene receptor antagonist, which can effectively improve airway inflammation in children with cough variant asthma (8). Budesonide is a glucocorticoid that enhances cell membrane stability, improves immune response, and relieves bronchial muscle spasms (9). Loratadine, a piperidine antihistamine commonly used in the treatment of allergic diseases, has also been used in recent years to treat cough variant asthma (10). In some related studies, montelukast sodium combined with budesonide or loratadine has been proven to have a good efficacy in the treatment of cough variant asthma $(11,12)$. However, few comparative studies have been made on the efficacy of these three drugs, montelukast sodium, budesonide and loratadine in cough variant asthma. In addition, tumor necrosis factor- $\alpha$ (TNF- $\alpha)$ and interleukin-4 (IL-4) are very important indicators of inflammation. Many studies have reported that the expression of these two factors in cough variant asthma was increased $(13,14)$.

This study retrospectively analyzed the medical records of 72 child patients with cough variant asthma and compared the clinical efficacy of montelukast sodium combined with budesonide or loratadine in cough variant asthma to provide reference in the drug treatment of cough variant asthma. 


\section{Patients and methods}

Research subjects. A retrospective analysis of the medical records of 72 children with cough variant asthma who were treated in Xuzhou Children's Hospital, Xuzhou Medical University (Xuzhou,China) from April 2015 to August 2017 was performed and the 72 children were divided into two groups: 35 children treated with montelukast sodium combined with budesonide in Group A, and 37 children treated with montelukast sodium combined with loratadine in Group B. Inclusion criteria were: Child patients that met the following diagnostic criteria (15): Cough, no dyspnea or wheezing, relieved symptoms after the inhalation of $\beta 2$-adrenergic receptor agonists, a positive result of bronchial hyperresponsiveness tested by methylcholine inhalation test or $\beta 2$ agonist inhalation test, aged from 3 to 14 years, no history of allergic diseases, no history of drug allergy, no history of respiratory diseases, no infectious disease. Exclusion criteria were: Children previously treated with leukotriene receptor antagonists, glucocorticoids, antihistamines; children with chronic cough; children with abnormal bleeding or coagulopathy combined with cardiovascular diseases; children complicated with digestive tract diseases; children who were transferred to another hospital halfway; children whose families did not cooperate with the treatment; children with incomplete medical records; children without complete 24-week follow-up data.

This study was approved by the Ethics Committee of Xuzhou Children's Hospital, Xuzhou Medical University, and the parents of the child patients signed informed consents.

Treatment plan. Patients in Group A were treated with montelukast sodium combined with budesonide, and patients in Group B were treated with montelukast sodium combined with loratadine. In both groups, patients under 6 years old were given montelukast sodium (Hangzhou MSD Pharmaceutical Co., Ltd., medical product permitted by the China Food and Drug Administration, code no. J20130047) $4.0 \mathrm{mg} / \mathrm{time}$ and once per day to chew before bedtime, while patients under 6 years old were given montelukast sodium $5.0 \mathrm{mg} / \mathrm{time}$ and once per day; in Group A, patients were given budesonide Aerosol (Shanghai Sine Pharmaceutical Laboratories Co., Ltd., medical product permitted by the China Food and Drug Administration, code no. H20010552) $1 \mathrm{mg} /$ time, once every 6-8 h; in Group B, patients under 30 kilograms were given loratadine (Jiangsu Hengrui Medicine Co., Ltd., medical product permitted by the China Food and Drug Administration, code no. H20020436) $5.0 \mathrm{mg} / \mathrm{time}$, while patients above 30 kilograms were given loratadine $10 \mathrm{mg} /$ time, once per day to take orally before bedtime. All the drug treatments lasted 12 weeks. Then the clinical efficacy was evaluated in terms of the lung function indexes and inflammatory markers before treatment and at 4 and 12 weeks after treatment. The adverse reactions, reoccurrence of disease and treatment compliance (the score of treatment compliance ranged from 0 to 100 points, graded by 3 doctors who were either chief physicians or had more senior titles, and a higher score indicated a better compliance) were also recorded.

Criteria of the efficacy evaluation. The evaluation of efficacy was divided into four degrees: Complete recovery, significant efficacy, certain efficacy, and no efficacy. Complete recovery means that the symptoms of cough and asthma totally disappeared with no complications, significant efficacy means that the symptoms of cough and asthma were basically improved, and the incidence rate of complications was $\leq 5 \%$; certain efficacy means that the symptoms of cough and asthma were partly improved, and the incidence rate of complications was $\leq 10 \%$; no efficacy refers to no improvement of symptoms and even worsen symptoms (12).

Detection of lung function indexes. The lung function indexes include the forced expiratory volume in the first second (FEV1), the ratio of the forced expiratory volume in the first second to the forced vital capacity (FEV1/FVC), and the peak expiratory flow (PEF). The Master Screen series lung function spirometer was purchased from Jaeger.

Detection of inflammatory markers. The inflammatory markers in this study included TNF- $\alpha$ and IL-4, which were measured by enzyme-linked immunosorbent assay. Operation guide for blood collection: The peripheral blood was collected by professional nurses in the morning and sent for examination within $1 \mathrm{~h}$. After centrifugation of the blood sample at $4,000 \times \mathrm{g}$ for $15 \mathrm{~min}$ at $4^{\circ} \mathrm{C}$, serum was taken to be tested in strict accordance with the manufacturer's instructions. All test kits were purchased from Shanghai Jingkang Biological Engineering Co., Ltd., and the article numbers of TNF- $\alpha$ and IL-4 detection kits were JLC7047 and JLC6419, respectively.

Detection of eosinophil granulocyte. The automatic hematology analyzer purchased from Beckman Coulter Commercial Enterprise (China) Co., Ltd. (article no. N/A) was used to detect the eosinophil granulocyte.

Detection of IgE. Enzyme-linked immunosorbent assay was used to detect IgE guided by the manufacturer's instructions. The detection kit for IgE was purchased from Shanghai Yanhui Biotechnology Co. Ltd. (article no. KA0216).

Statistical analysis. The software SPSS 19.0 (Asia Analytics Formerly SPSS China) was used. The enumeration data were expressed as $[\mathrm{n}(\%)]$, and the ratio was compared using the $\chi^{2}$ test. The measurement data were expressed with mean $\pm \mathrm{SD}$, and the t-test was used for comparison between the two groups. Comparison of different time-points within each group was performed with the repeated test of variance measurement. LSD test was used for post hoc test. Statistical significance was set at $\mathrm{P}<0.05$.

\section{Results}

General information. No significant difference in the sex ratio and age between the two groups existed as the Group A consisted of 17 males and 18 females, with an average age of $6.7 \pm 0.8$ years, and Group B consisted of 17 males and 20 females, with an average age of $6.5 \pm 0.7(\mathrm{P}>0.05)$. The height and weight of patients from the two groups were not statistically different $(\mathrm{P}>0.05)$. With regard to the duration of cough, proportion of eosinophils, proportion of basophils, and total serum IgE levels, the difference between the two groups was not statistically significant $(\mathrm{P}>0.05)$ (Table I). 
Table I. General information.

\begin{tabular}{|c|c|c|c|c|}
\hline Characteristics & Group A $(n=35)$ & Group B (n=37) & $\chi^{2} / \mathrm{t}$ value & P-value \\
\hline Sex & & & 0.050 & 0.824 \\
\hline Male & $17(48.57)$ & $17(45.95)$ & & \\
\hline Female & $18(51.43)$ & $20(54.05)$ & & \\
\hline Age (year) & $6.7 \pm 0.8$ & $6.5 \pm 0.7$ & 1.131 & 0.262 \\
\hline Height (cm) & $112.35 \pm 23.57$ & $118.42 \pm 24.73$ & 1.065 & 0.291 \\
\hline Weight (kg) & $26.13 \pm 4.32$ & $27.59 \pm 4.73$ & 1.365 & 0.177 \\
\hline Duration of cough (month) & $4.68 \pm 0.84$ & $4.75 \pm 0.92$ & 0.337 & 0.737 \\
\hline Eosinophils (\%) & $7.21 \pm 2.31$ & $7.56 \pm 2.62$ & 0.551 & 0.600 \\
\hline White blood cells $\left(10^{9} / 1\right)$ & $6.89 \pm 1.67$ & $7.02 \pm 1.83$ & 0.314 & 0.754 \\
\hline Neutrophil granulocytes (\%) & $58.75 \pm 8.96$ & $61.77 \pm 9.11$ & 1.417 & 0.161 \\
\hline Basophils (\%) & $0.083 \pm 0.034$ & $0.095 \pm 0.029$ & 1.614 & 0.111 \\
\hline Monocytes (\%) & $6.33 \pm 1.45$ & $6.41 \pm 1.62$ & 0.220 & 0.826 \\
\hline Lymphocytes (\%) & $32.75 \pm 9.62$ & $30.83 \pm 9.24$ & 0.864 & 0.391 \\
\hline Total serum IgE level (IU/ml) & $638.74 \pm 84.57$ & $613.85 \pm 78.69$ & 1.294 & 0.200 \\
\hline Score of the frequency of gastroesophageal reflux & $9.65 \pm 6.32$ & $10.12 \pm 6.96$ & 0.299 & 0.766 \\
\hline Airway hyper-reactivity & Positive & Positive & & \\
\hline Score of the childhood asthma control test & $16.34 \pm 2.13$ & $17.25 \pm 2.42$ & 1.690 & 0.096 \\
\hline Methacholine challenge test & Positive & Positive & & \\
\hline
\end{tabular}

Table II. Efficacy evaluation.

\begin{tabular}{|c|c|c|c|c|}
\hline Factors & Group A $(n=35)$ & Group B $(n=37)$ & $\chi^{2}$ value & P-value \\
\hline Complete recovery & $20(57.14)$ & $23(62.16)$ & 0.188 & 0.664 \\
\hline Significant efficacy & $10(28.57)$ & $9(24.32)$ & 0.167 & 0.683 \\
\hline Certain efficacy & $2(5.71)$ & $3(8.11)$ & 0.159 & 0.690 \\
\hline No efficacy & $3(8.57)$ & $2(5.41)$ & 0.279 & 0.597 \\
\hline Overall effective rate & $32(91.43)$ & 35 (94.59) & 0.279 & 0.597 \\
\hline
\end{tabular}

Evaluation of efficacy. The evaluation of the efficacy of the two groups at the 12 week after treatment showed no significant difference in the rate of complete recovery, the rate of significant efficacy, the rate of certain efficacy, the rate of no efficacy and the total effective rate between the two groups (P>0.05) (Table II).

Changes in lung function indexes in the two groups. The results of lung function tests in the two groups showed no significant difference in the FEV1, FEV1/FVC and PEF between the two groups at three time-points: Before treatment, the 4th week after treatment, and the 12th week after treatment $(\mathrm{P}>0.05)$. According to the comparison within each group, the levels of FEV1, FEV1/FVC and PEF at the 4th and 12th week after treatment were higher than those before treatment (both $\mathrm{P}<0.05$ ), and the levels of FEV1, FEV1/FVC and PEF at the 12th week after treatment in the two groups were higher than those at the 4th week after treatment (both $\mathrm{P}<0.05$ ) (Fig. 1).

Changes in inflammatory markers in the two groups. The tests of the inflammatory markers in the two groups showed that there was no significant difference in the TNF- $\alpha$ and IL-4 between the two groups at three time-points: Before treatment, the 4th week after treatment, and the 12th week after treatment ( $\mathrm{P}>0.05)$. According to the comparison within each group, the TNF- $\alpha$ and IL-4 levels were significantly decreased after treatment and the inflammatory reaction was under effective control. The TNF- $\alpha$ and IL-4 levels at the 4th and 12th week after treatment were lower than those before treatment (both $\mathrm{P}<0.05)$, and the levels of TNF- $\alpha$ and IL- 4 at the 12 th week after treatment in the two groups were lower than those at the 4th week after treatment (both $\mathrm{P}<0.05$ ) (Fig. 2).

Changes in the eosinophil granulocytes in the two groups. In both groups, the eosinophil granulocyte levels at three time-points (before treatment, at the 4th week after treatment, and at the 12th week after treatment) were not statistically different $(\mathrm{P}>0.05)$. According to the comparison within each group, no statistical difference was detected between the eosinophil granulocyte level before treatment and the eosinophil granulocyte level at the 4th week after treatment $(\mathrm{P}>0.05)$, but the eosinophil granulocyte level at the 12 th week after treatment was lower than that at the 4 th week after treatment $(\mathrm{P}<0.05)$ (Table III). 

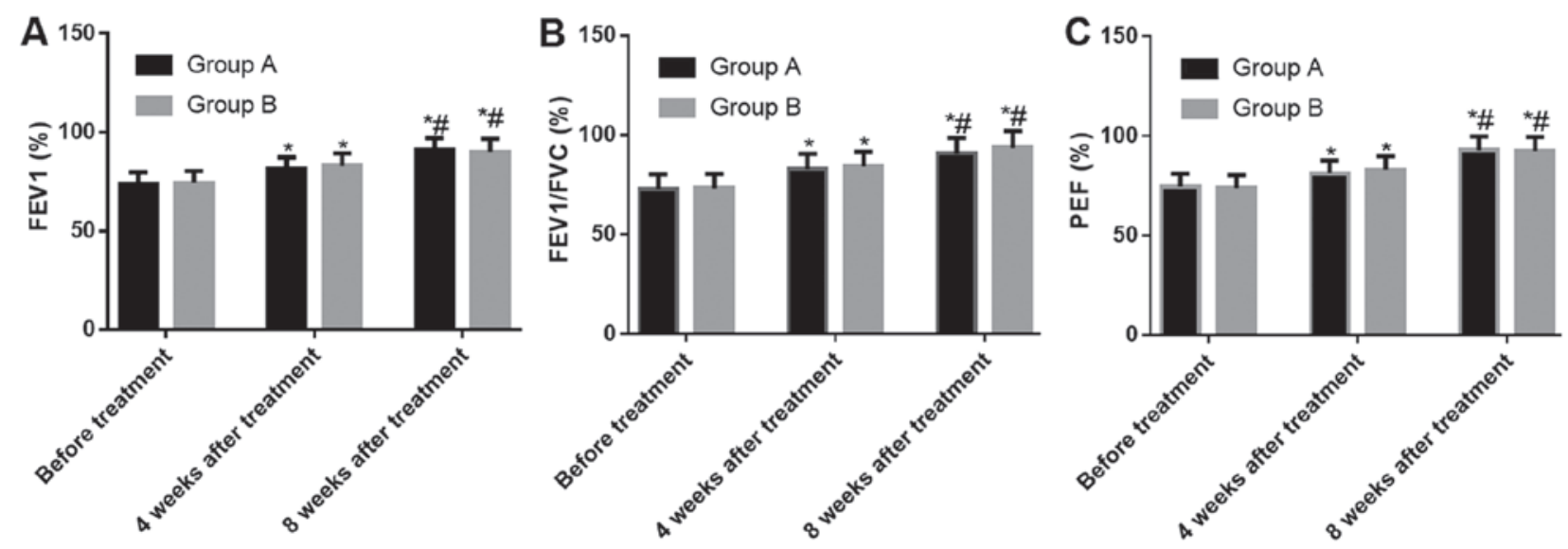

Figure 1. Changes in lung function indexes in the two groups. (A) Changes of FEV1 before and after treatment; (B) Changes of FEV1/FVC before and after treatment; $(\mathrm{C})$ Changes of PEF before and after treatment." $\mathrm{P}<0.05$, compared with the same group before treatment; " $\mathrm{P}<0.05$, compared with the same group 4 weeks after treatment. FEV1, forced expiratory volume in the first second; FEV1/FVC, forced expiratory volume in the first second to the forced vital capacity; PEF, peak expiratory flow.
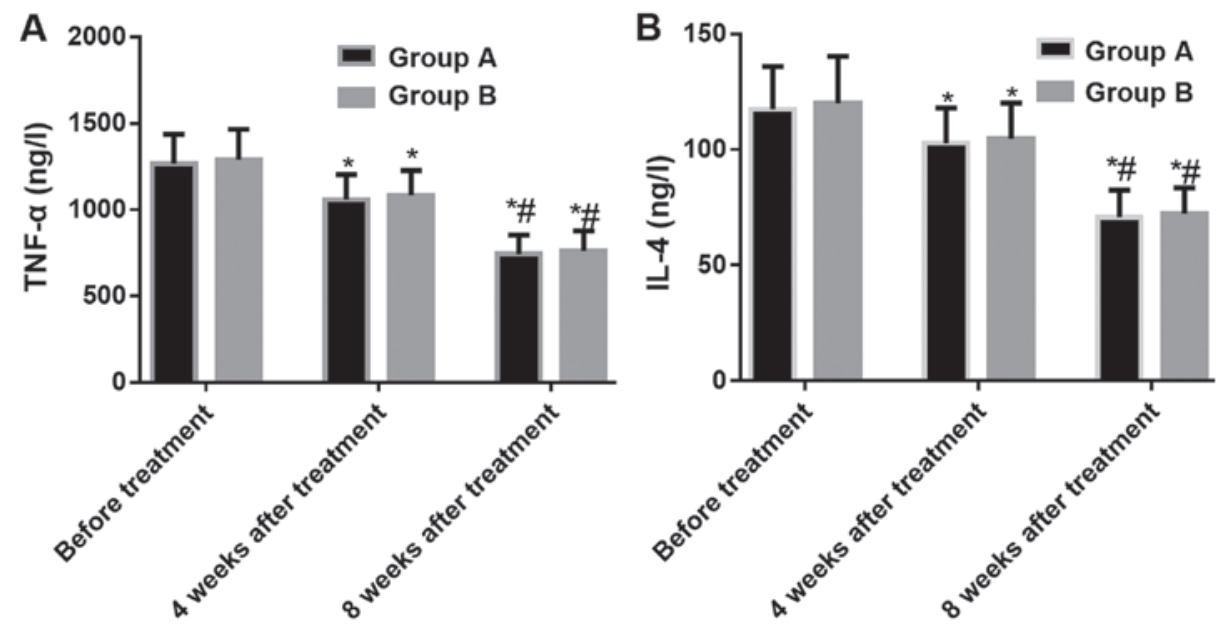

Figure 2. Changes in inflammatory markers in the two groups. (A) Changes of TNF- $\alpha$ before and after treatment; (B) Changes of IL-4 before and after treatment. " $\mathrm{P}<0.05$, compared with the same group before treatment; ${ }^{~} \mathrm{P}<0.05$, compared with the same group 4 weeks after treatment. TNF- $\alpha$, tumor necrosis factor- $\alpha$; IL-4, interleukin- 4 .

Changes in the IgE in the two groups. The tests of the IgE in the two groups showed that there was no significant difference in the IgE levels between the two groups at three time-points: before treatment, at the 4 th week after treatment, and at the 12th week after treatment $(\mathrm{P}>0.05)$. According to the comparison within each group, the IgE levels at the 4th and 12th week after treatment were lower than those before treatment (both $\mathrm{P}<0.05)$, and the IgE levels at the 12th week after treatment in the two groups were lower than those at the 4th week after treatment (both $\mathrm{P}<0.05$ ) (Table IV).

The incidence of adverse reactions in the two groups. The two groups had 3 cases each of adverse reactions (not very severe) which were healed after receiving the corresponding treatment intervention. No statistically significant difference in the incidence of complications and the incidence of respiratory tract infection between the two groups was found as there was 1 case of respiratory tract infection, 1 case of pneumonia, and 1 case of drowsiness in Group A, while 2 cases of respiratory tract infection and 1 case of fatigue in Group B (both $\mathrm{P}>0.05$ ) (Table V).
Recurrence of the disease in the two groups. The follow-up continued for 24 weeks after the completion of treatment. During the follow-up, 6 patients in Group A had recurrence, with a recurrence rate of $17.14 \%$, and 7 patients in Group B had recurrence, with a recurrence rate of $18.92 \%$, the difference between the two groups was not statistically significant $(\mathrm{P}>0.05)$ (Fig. 3).

Analysis of the treatment compliance. The treatment compliance analysis of the two groups showed that the treatment compliance score of Group A $(80.42 \pm 21.75)$ was significantly lower than that of Group B $(91.36 \pm 16.78)(\mathrm{P}<0.05)$ (Fig. 4).

\section{Discussion}

Considering the fact that cough variant asthma has similar clinical symptoms to clinical typical asthma such as respiratory remodeling, inflammatory cell infiltration and generally airway hyperresponsiveness, a consensus has been reached worldwide that the principles of treating cough variant asthma 
Table III. Changes in the eosinophil granulocytes in the two groups (\%).

\begin{tabular}{lcrr}
\hline Time-points & Group A (n=35) & Group B (n=37) & t value \\
\hline Before treatment & $7.21 \pm 2.31$ & $7.56 \pm 2.62$ & 0.551 \\
The 4th week after treatment & $6.86 \pm 1.14$ & $6.75 \pm 1.17$ & 0.600 \\
The 12th week after treatment & $6.32 \pm 0.97^{\mathrm{a}, \mathrm{b}}$ & $6.44 \pm 0.85^{\mathrm{a}, \mathrm{b}}$ & 0.404 \\
\end{tabular}

${ }^{\mathrm{a}} \mathrm{P}<0.05$, when compared with the data before treatment in the same group; ${ }^{\mathrm{b}} \mathrm{P}<0.05$, when compared with the data at the 4 th week after treatment in the same group.

Table IV. Changes in the IgE in the two groups (IU/ml).

\begin{tabular}{llrr}
\hline Time-points & Group A $(\mathrm{n}=35)$ & Group B (n=37) & t value \\
\hline Before treatment & $638.74 \pm 84.57$ & $613.85 \pm 78.69$ & 1.294 \\
The 4th week after treatment & $582.64 \pm 70.51^{\mathrm{a}}$ & $574.35 \pm 70.51^{\mathrm{a}}$ & 0.200 \\
The 12th week after treatment & 495.22 after $^{\mathrm{a}, \mathrm{b}}$ & 483.87 after $^{\mathrm{a}, \mathrm{b}}$ & 0.506 \\
\hline
\end{tabular}

${ }^{\mathrm{a}} \mathrm{P}<0.05$, when compared with the data before treatment in the same group; ${ }^{\mathrm{b}} \mathrm{P}<0.05$, when compared with the data at the 4 th week after treatment in the same group.

Table V. The incidence of adverse reactions in the two groups of children.

\begin{tabular}{lll}
\hline Factors & $\begin{array}{c}\text { Group A } \\
(\mathrm{n}=35)\end{array}$ & $\begin{array}{c}\text { Group B } \\
(\mathrm{n}=37)\end{array}$ \\
\hline Respiratory tract infection & $1(2.86)$ & $2(5.41)$ \\
Pneumonia & $1(2.86)$ & $0(0.00)$ \\
Fatigue & $0(0.00)$ & $1(2.70)$ \\
Drowsiness & $1(2.86)$ & $0(0.00)$ \\
Overall incidence rate & $3(8.57)$ & $3(8.11)$ \\
\hline
\end{tabular}

are consistent with that of treating typical asthma $(15,16)$. However, different countries or regions lack a unified opinion on the choice of therapeutic drugs, and there is no relevant evidence-based medical basis for the choice of treatment options. This study retrospectively analyzed the efficacy of montelukast sodium combined with budesonide or loratadine in cough variant asthma in order to provide a reference for clinical treatment.

The study collected the medical records of two groups of children with cough variant asthma strictly according to the inclusion and exclusion criteria. No statistical difference between the two groups of patients existed in the general data, indicating that the two groups were comparable in this study and the results of this study had certain credibility. Leukotriene receptor antagonists, glucocorticoids, and antihistamines are the three major types of drugs for the treatment of cough variant asthma (17,18). Leukotriene can induce spasms of bronchial smooth muscle, increase of vascular permeability and infiltration of inflammatory cells. Montelukast sodium, one of the most frequently mentioned leukotriene receptor antagonists in the treatment of cough variant asthma, is reported worldwide

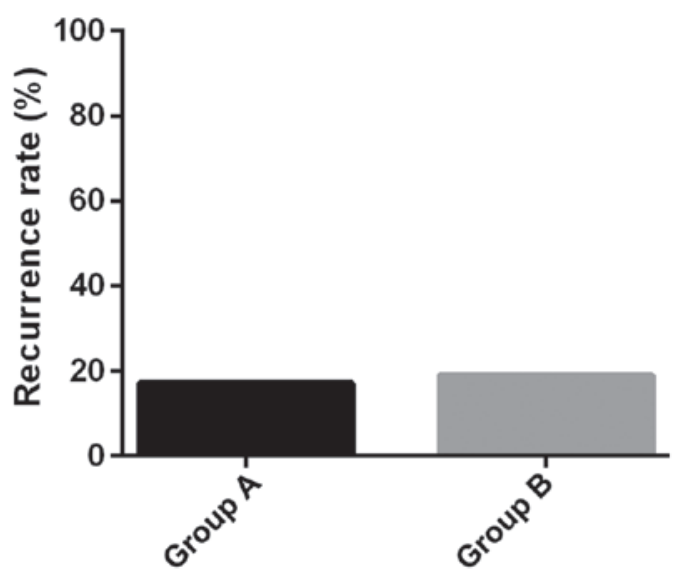

Figure 3. Recurrence in the two groups. The recurrence rates of the two groups were not statistically different as Group A had a recurrence rate of $17.14 \%$ and Group B had a recurrence rate of $18.92 \%(\mathrm{P}>0.05)$

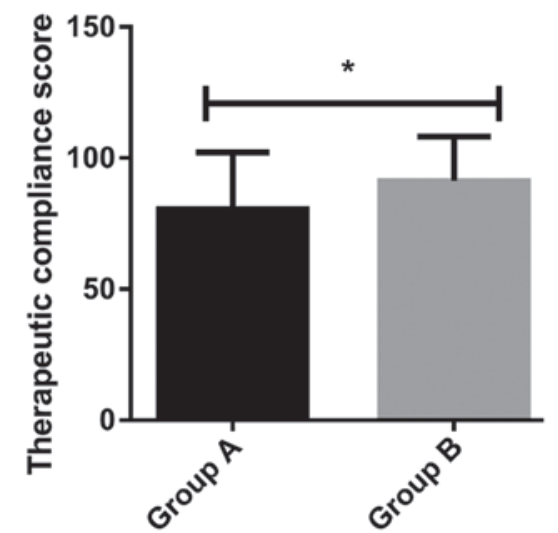

Figure 4. Analysis of the treatment compliance. The treatment compliance scores of Group A were significantly lower than those of Group B $(\mathrm{P}<0.05)$. ${ }^{*} \mathrm{P}<0.05$, when Group A was compared with Group B. 
with excellent efficacy, low adverse reactions and high patient compliance $(8,19)$. Studies have reported that the main cause of cough variant asthma is airway hyperresponsiveness caused by abnormal inflammatory reactions which can be effectively improved by glucocorticoids such as budesonide, especially through the dosing method of atomization inhalation $(20,21)$. Loratadine, a piperidine antihistamine that antagonizes peripheral $\mathrm{H} 1$ receptors over a long time efficiently, can greatly improve the airway hyperresponsiveness, inhibit the degranulation of eosinophils and mast cells, and reduce the release of leukotrienes (22). The good complementarity and synergistic therapeutic effects of montelukast sodium combined with budesonide or loratadine in cough variant asthma have been reported in many studies $(11,12)$. According to the results of this study, both methods of drug treatments had a good therapeutic effect with an effective rate of $>90 \%$, and improved eosinophil granulocyte level and $\operatorname{IgE}$ level, similarly to previous research results $(11,12)$. The two methods of drug treatment both had similar efficacy in improving the lung function indexes and the inflammation symptoms, slight adverse reactions, and not statistically different incidence rates of recurrence, proving that montelukast sodium combined with budesonide or loratadine were equivalent in the treatment efficacy of cough variant asthma. However, the atomization inhalation therapy's requiring special inhalation techniques and shortage of aerosol devices usually causes low compliance in patients $(23,24)$ which was reflected in this study. Therefore, for younger patients with poor compliance, montelukast sodium combined with loratadine can be a better choice.

However, this study also had some defects. In this study, we did not find any differences between the two combination therapies, which may be related to our exclusion criteria or the number of our cases, or due to the 12 weeks of treatment time, while the follow-up time was 24 weeks after the completion of the treatment. Both were relatively short. The long-term efficacy of the two treatment methods still needs to be further studied and determined, and continued follow-up will be needed in the future. Also, the age range of the patients included in this study was large, and because TNF- $\alpha$ and IL-4 are the most commonly detected inflammatory factors in our hospital, in order to avoid the occurrence of fewer cases, we did not include other inflammatory indicators, such as IL-5, IL-13, which might lead to bias in the results of compliance analysis, so more clinical data and narrowed age range will be asked in future studies to make further analysis. This study aimed to inspire more scholars to conduct large-scale, multi-center experiments for in-depth research.

In summary, both the combination of montelukast sodium and budesonide and the combination of montelukast sodium and loratadine had similar efficacy in cough variant asthma to effectively improve the lung function and inflammatory response in patients without causing more adverse reactions and higher recurrence rate, both worthy of clinical promotion. For patients with younger age and poor compliance, the method of using montelukast sodium combined with loratadine is preferably advised.

\section{Acknowledgements}

Not applicable.

\section{Funding}

No funding was received.

\section{Availability of data and materials}

The datasets used and/or analyzed during the present study are available from the corresponding author on reasonable request.

\section{Authors' contributions}

HW and WL helped with detection of lung function indexes. HW and ZJ worked on detection of inflammatory markers. XX and GQ collected, analyzed and interpreted the general data of patients, and revised the manuscript critically for important intellectual content. HW was involved in the writing of this manuscript. All the authors read and approved the final manuscript.

\section{Ethics approval and consent to participate}

The study was approved by the Ethics Committee of Xuzhou Children's Hospital, Xuzhou Medical University (Xuzhou, China). Signed informed consents were obtained from the parents of the child patients.

\section{Patient consent for publication}

Not applicable.

\section{Competing interests}

The authors declare that they have no competing interests.

\section{References}

1. Hossain SS, Islam MS, Rahman MM, Dey S and Mahmud K: Clinical and demographic profiles of patients diagnosed as cough variant asthma attended at Tertiary Referral Hospital. JNINB 2: $1,2016$.

2. Kanemitsu Y, Niimi A, Matsumoto H, Iwata T, Ito I, Oguma T, Inoue H, Tajiri T, Nagasaki T, Izuhara Y, et al: Gastroesophageal dysmotility is associated with the impairment of cough-specific quality of life in patients with cough variant asthma. Allergol Int 65: 320-326, 2016

3. Tagaya E, Kondo M, Kirishi S, Kawagoe M, Kubota N and Tamaoki J: Effects of regular treatment with combination of salmeterol/fluticasone propionate and salmeterol alone in cough variant asthma. J Asthma 52: 512-518, 2015.

4. Tajiri T, Niimi A, Matsumoto H, Ito I, Oguma T, Otsuka K, Takeda T, Nakaji H, Inoue H, Iwata T, et al: Prevalence and clinical relevance of allergic rhinitis in patients with classic asthma and cough variant asthma. Respiration 87: 211-218, 2014.

5. Evans DJ, Taylor DA, Zetterstrom O, Chung KF, O'Connor BJ and Barnes PJ: A comparison of low-dose inhaled budesonide plus theophylline and high-dose inhaled budesonide for moderate asthma. N Engl J Med 337: 1412-1418, 1997.

6. Bao W, Chen Q, Lin Y, Liu H, Zhao G, Chen Z and Zhou X: Efficacy of procaterol combined with inhaled budesonide for treatment of cough-variant asthma. Respirology 18 (Suppl 3): 53-61, 2013

7. Frølund L: Efficacy of an oral antihistamine, loratadine, as compared with a nasal steroid spray, beclomethasone dipropionate, in seasonal allergic rhinitis. Clin Otolaryngol Allied Sci 16: 527-531, 1991.

8. Wang X, Liu B, Lu B, Zhang Y, Wang L, Li H, Han X and Ding D: Micro-invasive embedding combined with montelukast sodium for children cough variant asthma: A randomized controlled trial. Zhongguo Zhen Jiu 37: 259-264, 2017 (In Chinese). 
9. Zhou X, Hong J, Cheng H, Xie J, Yang J, Chen Q, He S, Li Y, Zhou X and Li C: Budesonide suspension nebulization treatment in Chinese pediatric patients with cough variant asthma: A multi-center observational study. J Asthma 53: 532-537, 2016.

10. Steffey EP, Mama KR, Galey FD, Puschner B and Woliner MJ: Effects of sevoflurane dose and mode of ventilation on cardiopulmonary function and blood biochemical variables in horses. Am J Vet Res 66: 606-614, 2005.

11. Wei Y, Li DS, Liu JJ, Zhang J and Zhao HE: Therapeutic effect and safety of montelukast sodium combined with budesonide in children with cough variant asthma: A meta analysis. Zhongguo Dang Dai Er Ke Za Zhi 18: 1100-1105, 2016 (In Chinese).

12. Wang XP, Yang LD and Zhou JF: Montelukast and budesonide combination for children with chronic cough-variant asthma. Medicine (Baltimore) 97: e11557, 2018.

13. Xiao Q and Qin X: Effects of salmeterol xinafoate and fluticasone propionate powder in combination with montelukast on pulmonary function and serum inflammatory cytokines in children with cough variant asthma. Zhongguo Jiceng Yiyao 25: 182-185, 2018 (In Chinese).

14. Jiao HY, Su WW, Li PB, Liao Y, Zhou Q, Zhu N and He LL: Therapeutic effects of naringin in a guinea pig model of ovalbumin-induced cough-variant asthma. Pulm Pharmacol Ther 33: 59-65, 2015.

15. Takemura M, Niimi A, Matsumoto $H$, Ueda T, Yamaguchi M, Matsuoka H, Jinnai M, Chin K and Mishima M: Atopic features of cough variant asthma and classic asthma with wheezing. Clin Exp Allergy 37: 1833-1839, 2007.

16. Saito N, Itoga M, Tamaki M, Yamamoto A and Kayaba H: Cough variant asthma patients are more depressed and anxious than classic asthma patients. J Psychosom Res 79: 18-26, 2015.

17. Dicpinigaitis PV: Chronic cough due to asthma: ACCP evidencebased clinical practice guidelines. Chest 129 (Suppl 1): 75S-79S, 2006.
18. Usta Guc B, Asilsoy S and Durmaz C: The assessment and management of chronic cough in children according to the British Thoracic Society guidelines: Descriptive, prospective, clinical trial. Clin Respir J 8: 330-337, 2014.

19. Matsuse H and Kohno S: Leukotriene receptor antagonists pranlukast and montelukast for treating asthma. Expert Opin Pharmacother 15: 353-363, 2014.

20. Sun LH, Chen AH and Zhang Y: Therapeutic efficacy and follow-up study of inhaled corticosteroids vs. oral montelukast in treatment of cough variant asthma. Zhonghua Er Ke Za Zhi 46: 85-88, 2008 (In Chinese)

21. Peng QF and Kong LF: The levels of nerve growth factor and IL-4 in induced sputum and characteristics of airway inflammation in cough variant asthma. Zhonghua Nei Ke Za Zhi 50: 221-224, 2011 (In Chinese)

22. Fujimura M, Ogawa H, Nishizawa $Y$ and Nishi K: Comparison of atopic cough with cough variant asthma: Is atopic cough a precursor of asthma? Thorax 58: 14-18, 2003.

23. Sanchis J, Gich I and Pedersen S; Aerosol Drug Management Improvement Team (ADMIT): Systematic review of errors in inhaler use: Has patient technique improved over time? Chest 150: 394-406, 2016.

24. Deuse T, Hua X, Stubbendorff M, Spin JM, Neofytou E, Taylor V, Chen Y, Park G, Fink JB, Renne T, et al: The selective JAK1/3-inhibitor R507 mitigates obliterative airway disease both with systemic administration and aerosol inhalation. Transplantation 100: 1022-1031, 2016.

(i) (2) This work is licensed under a Creative Commons Attribution-NonCommercial-NoDerivatives 4.0 International (CC BY-NC-ND 4.0) License. 